J. Austral. Math. Soc. 22 (Series A) (1976), 54-64.

\title{
ON THE REDUCTION OF POSITIVE QUATERNARY QUADRATIC FORMS
}

\author{
Dedicated to George Szekeres on his 65th birthday \\ E. S. BARNES and M. J. COHN
}

(Received 26 June, 1975)

Communicated by Jane Pitman

\begin{abstract}
A fundamental region for the reduction of positive quaternary quadratic forms is exhibited. It is a convex polyhedral cone with twelve edges in the 10-dimensional space of quaternary quadratic forms.
\end{abstract}

\section{Introduction}

Two positive definite $n$-ary quadratic forms $f(x)=x^{\prime} A \boldsymbol{x}$ and $g(x)=x^{\prime} B \boldsymbol{x}$, where $A, B$ are symmetric, are said to be equivalent (written $f \sim g$ ) if there exists an integral unimodular matrix $T$ for which $B=T^{\prime} A T$. The basic problem of the reduction theory of positive quadratic forms is to specify a region $D$ (called a fundamental region) in the $\frac{1}{2} n(n+1)$-dimensional coefficient space of forms satisfying

(i) for every positive definite $n$-ary quadratic form $f$, there exists a form $f_{0} \in D$ with $f_{0} \sim f$ and

(ii) if $f, g$ are distinct forms in the interior of $D$, then $f \not f$.

It is desirable if, as for the well-known reduction methods for $\boldsymbol{n}=\mathbf{2}$ and $n=3, D$ is a convex polyhedral cone. Minkowski (1905) and Venkov (1940) established methods for the construction of such fundamental regions for all $n$; however, when $n \geqq 4$, it is difficult to describe these explicitly, and they have

* The authors acknowledge gratefully the financial support of the Australian Research Grants Committee. 
very large numbers of facets and edges. For $n=4$, Štogrin (1974) produced a fundamental region which is the union of three cones, but which is not convex; here we specify a fundamental region which is a convex cone with only twelve edges.

We show:

A fundamental region of positive quaternary quadratic forms is given by the convex cone of forms which is the set of non-negative linear combinations of the following twelve forms:

$$
\begin{aligned}
& x_{1}^{2}+x_{2}^{2}, x_{2}^{2}, x_{3}^{2}, x_{4}^{2},\left(x_{1}-x_{3}\right)^{2},\left(x_{1}-x_{4}\right)^{2}, \\
& \left(x_{2}-x_{3}\right)^{2}+\left(x_{2}-x_{4}\right)^{2},\left(x_{2}-x_{4}\right)^{2}, x_{3}^{2}+x_{4}^{2}+\left(x_{3}-x_{4}\right)^{2}, \\
& \omega_{0}(x)=\sum_{0 \leqq i<j \leqq 4}\left(x_{i}-x_{i}\right)^{2} \quad\left(\text { where } x_{0} \equiv 0\right), \\
& \omega_{1}(x)=4\left\{x_{1}^{2}+x_{2}^{2}+x_{3}^{2}+x_{4}^{2}+x_{1} x_{2}-x_{1} x_{3}-x_{1} x_{4}-x_{2} x_{3}-x_{2} x_{4}\right\}
\end{aligned}
$$

and

$$
\begin{aligned}
\alpha(x)=x_{1}^{2}+x_{2}^{2} & +x_{3}^{2}+x_{4}^{2}+\left(x_{1}-x_{3}\right)^{2}+\left(x_{1}-x_{4}\right)^{2} \\
& +\left(x_{2}-x_{3}\right)^{2}+\left(x_{2}-x_{4}\right)^{2}+\left(x_{1}+x_{2}-x_{3}-x_{4}\right)^{2} .
\end{aligned}
$$

We prove this by a refinement of the reduction into the cones $R\left(\varphi_{0}\right)$ and $R\left(\varphi_{1}\right)$ of the first reduction method of Voronoi (1907). We note that $\omega_{0}$ and $\omega_{1}$ are respectively (multiples of) the forms adjoint to the perfect forms

$$
\varphi_{0}(x)=\sum_{1 \leq i \leq j \leq 4} x_{i} x_{j}, \quad \varphi_{1}(x)=\varphi_{0}(x)-x_{1} x_{2} .
$$

\section{Some preliminaries}

For the proof we require some properties of Voronoi reduction. Consider the transformations

$$
\mathscr{T}: A \mapsto T^{\prime} A T
$$

where $T$ is an integral unimodular matrix. We note that such a transformation is linear and therefore continuous. Voronoi's reduction method partitions the space of forms into cones $R(\varphi)$, where $\varphi$ is perfect. A transformation $\mathscr{T}$ either leaves a cone $R(\varphi)$ invariant, or transforms it into a cone $R\left(\varphi^{\prime}\right)$ which has no interior form in common with $R(\varphi)$. So, if two distinct forms $f, g \in$ int $R(\varphi)$ are equivalent, then there exists an automorphism of $R(\varphi)$ transforming $f$ to $g$.

It is frequently convenient to identify the transformations of the space of forms with the corresponding transformations of $n$-dimensional space. How- 
ever, since both $T$ and $-T$ correspond to the same transformation $\mathscr{T}$ in (2.1), it is sometimes convenient to remove the factor $\{ \pm I\}$ in specifying the group $\mathscr{A}(\varphi)$ of automorphisms of a region $R(\varphi)$.

For $n=4$, any positive definite quadratic form is equivalent to a form of $R\left(\varphi_{0}\right)$ or $R\left(\varphi_{1}\right)$. The automorphism groups of $\varphi_{0}$ and $\varphi_{1}$ are described by Coxeter (1951), where these forms are denoted by $A_{4}$ and $B_{4}$.

\section{Reduction of $R\left(\varphi_{0}\right)$}

The cone $R\left(\varphi_{0}\right)$ of Voronoi's reduction contains precisely those forms $f$ of the type

$$
f(x)=\sum_{0 \leqq i<j \leqq 4} \rho_{i j}\left(x_{i}-x_{j}\right)^{2}
$$

where $x_{0} \equiv 0$ and $\rho_{i j} \geqq 0$ for all $i, j$. The group of automorphisms of $R\left(\varphi_{0}\right)$ (after removing the factor $\{ \pm I\}$ ) may be identified with the group of permutations of $x_{0}, x_{1}, \cdots, x_{n}$.

Since two forms $f, g$ in the interior of $R\left(\varphi_{0}\right)$ are equivalent only if there exists an automorphism of $R\left(\varphi_{0}\right)$ transforming $f$ to $g$, we use the group of automorphisms of $\boldsymbol{R}\left(\varphi_{0}\right)$ to obtain a fundamental region for those forms in $R\left(\varphi_{0}\right)$. Firstly, by a suitable permutation of $x_{0}, x_{1}, \cdots, x_{n}$, we may insist that

$$
\rho_{12}=\min _{0 \leq i<j \leq 4} \rho_{i j}
$$

This divides the variables into the two sets $\left\{x_{1}, x_{2}\right\}$ and $\left\{x_{0}, x_{3}, x_{4}\right\}$. To distinguish $x_{0}$ from $x_{3}$ and $x_{4}$, by permutation of $x_{0}, x_{3}$ and $x_{4}$, we may insist further that

$$
\rho_{34}=\min \left\{\rho_{03}, \rho_{04}, \rho_{34}\right\} \text {. }
$$

The variables $x_{1}$ and $x_{2}$ are distinguished by arranging that

$$
\rho_{01}=\min \left\{\rho_{01}, \rho_{02}\right\} \text {. }
$$

The only variables not fully distinguished yet are $x_{3}$ and $x_{4}$. Therefore we stipulate that

$$
\rho_{23}=\min \left\{\rho_{23}, \rho_{24}\right\} \text {. }
$$

The conditions (3.2), (3.3), (3.4) and (3.5) define a convex polyhedral cone in the 10-dimensional coefficient space of forms with edge-forms

$$
\begin{gathered}
x_{1}^{2}+x_{2}^{2}, x_{2}^{2}, x_{3}^{2}, x_{4}^{2},\left(x_{1}-x_{3}\right)^{2},\left(x_{1}-x_{4}\right)^{2}, \\
\left(x_{2}-x_{3}\right)^{2}+\left(x_{2}-x_{4}\right)^{2},\left(x_{2}-x_{4}\right)^{2}, x_{3}^{2}+x_{4}^{2}+\left(x_{3}-x_{4}\right)^{2}
\end{gathered}
$$


and

$$
\omega_{0}(x)=\sum_{0 \leqq i<j \leq 4}\left(x_{i}-x_{j}\right)^{2} \quad\left(\text { where } x_{0} \equiv 0\right) .
$$

Let us denote this region by $F$. Clearly any form of the type (3.1) is equivalent to a form of $F$. Furthermore, a form $f$ is in the interior of $F$ if and only if each of (3.2), (3.3), (3.4) and (3.5) distinguish the relevant $x_{i}$ uniquely, and so $F$ must be a fundamental region for forms equivalent to those in $R\left(\varphi_{0}\right)$.

\section{Reduction of $R\left(\varphi_{1}\right)$}

The set $R\left(\varphi_{1}\right)$ is the convex polyhedral cone with edge-forms

$$
\begin{gathered}
x_{1}^{2}, x_{2}^{2}, x_{3}^{2}, x_{4}^{2},\left(x_{1}-x_{3}\right)^{2},\left(x_{1}-x_{4}\right)^{2},\left(x_{2}-x_{3}\right)^{2}, \\
\left(x_{2}-x_{4}\right)^{2},\left(x_{3}-x_{4}\right)^{2},\left(x_{1}+x_{2}-x_{3}\right)^{2},\left(x_{1}+x_{2}-x_{4}\right)^{2} \text { and }\left(x_{1}+x_{2}-x_{3}-x_{4}\right)^{2} .
\end{gathered}
$$

The cone is more easily examined after making the transformation

so that

$$
\begin{aligned}
& 2 X_{1}=x_{1}+x_{2} \\
& 2 X_{2}=x_{1}-x_{2} \\
& 2 X_{3}=-x_{1}-x_{2}+2 x_{3} \\
& 2 X_{4}=-x_{1}-x_{2}+2 x_{4},
\end{aligned}
$$

$$
\begin{array}{ll}
X_{1}-X_{2}=x_{2} & X_{1}+X_{2}=x_{1} \\
X_{1}-X_{3}=x_{1}+x_{2}-x_{3} & X_{1}+X_{3}=x_{3} \\
X_{1}-X_{4}=x_{1}+x_{2}-x_{4} & X_{1}+X_{4}=x_{4} \\
X_{2}-X_{3}=x_{1}-x_{3} & X_{2}+X_{3}=-x_{2}+x_{3} \\
X_{2}-X_{4}=x_{1}-x_{4} & X_{2}+X_{4}=-x_{2}+x_{4} \\
X_{3}-X_{4}=x_{3}-x_{4} & X_{3}+X_{4}=-x_{1}-x_{2}+x_{3}+x_{4} .
\end{array}
$$

Then the forms of $R\left(\varphi_{1}\right)$ are precisely those forms $f$ with

$$
f(x)=\sum_{1 \leq i<j \leq 4} \sigma_{i j}\left(X_{i}-X_{j}\right)^{2}+\sum_{1 \leq i<j \leq 4} \tau_{i j}\left(X_{i}+X_{j}\right)^{2}
$$

where $\sigma_{i j}, \tau_{i j} \geqq 0$ for all $i, j$. In particular, the adjoint form of $\varphi_{1}$ is now

$$
\omega_{1}(x)=\sum_{i=1}^{4} X_{i}^{2}=\frac{1}{6}\left\{\sum_{1 \leq i<j \leq 4}\left(X_{i}-X_{i}\right)^{2}+\sum_{1 \leq i<j \leq 4}\left(X_{i}+X_{j}\right)^{2}\right\} .
$$


Similarly to the reduction of $R\left(\varphi_{0}\right)$, we make use here of the automorphism group $\mathscr{A}\left(\varphi_{1}\right)$ of $R\left(\varphi_{1}\right)$. Coxeter (1951) showed that this group (including $\pm I$ ) has order $3 \cdot 2^{4} \cdot 4 !=1152$; it is generated by

(i) the permutations of $X_{1}, X_{2}, X_{3}, X_{4}$,

(ii) arbitrary changes of sign of the $X_{i}$ and

(iii) the transformation

$$
U: 2 X_{i} \mapsto 2 X_{i}-\sum_{j=1} X_{i} \cdot(i=1,2,3,4),
$$

for which

and

$$
X_{i}-X_{j} \mapsto X_{i}-X_{j}
$$

$$
X_{i}+X_{j} \mapsto-\left(X_{k}+X_{l}\right),
$$

where $i, j, k, l$ is any arrangement of $1,2,3,4$.

Now suppose $f$ is a given form of $R\left(\varphi_{1}\right)$, expressed in the form (4.1). By applying permutations and changes of sign to the $X_{i}$, we may arrange that

$$
\sigma_{13}=\min _{1 \leqq i<j \leqq 4}\left\{\sigma_{i j}, \tau_{i j}\right\}
$$

Further, we may require that $\sigma_{14}$ is the least coefficient with one subscript 1 or 3 , that is,

$$
\sigma_{14}=\min \left\{\sigma_{12}, \tau_{12}, \sigma_{14}, \tau_{14}, \sigma_{23}, \tau_{23}, \sigma_{34}, \tau_{34}\right\} .
$$

These two conditions give

$$
\begin{array}{ll}
\sigma_{12}=\sigma_{13}{ }^{\prime}+\sigma_{14^{\prime}}+\sigma_{12}{ }^{\prime} & \tau_{12}=\sigma_{13}{ }^{\prime}+\sigma_{14}{ }^{\prime}+\tau_{12}{ }^{\prime} \\
\sigma_{13}=\sigma_{13}{ }^{\prime} & \tau_{13}=\sigma_{13}{ }^{\prime}+\tau_{13}{ }^{\prime} \\
\sigma_{14}=\sigma_{13}{ }^{\prime}+\sigma_{14^{\prime}} & \tau_{14}=\sigma_{13}{ }^{\prime}+\sigma_{14}{ }^{\prime}+\tau_{14}{ }^{\prime} \\
\sigma_{23}=\sigma_{13}{ }^{\prime}+\sigma_{14^{\prime}}+\sigma_{23}{ }^{\prime} & \tau_{23}=\sigma_{13}{ }^{\prime}+\sigma_{14}{ }^{\prime}+\tau_{23}{ }^{\prime} \\
\sigma_{24}=\sigma_{13}{ }^{\prime}+\sigma_{24^{\prime}} & \tau_{24}=\sigma_{13}{ }^{\prime}+\tau_{24^{\prime}} \\
\sigma_{34}=\sigma_{13}{ }^{\prime}+\sigma_{14}{ }^{\prime}+\sigma_{34^{\prime}} & \tau_{34}=\sigma_{13}{ }^{\prime}+\sigma_{14}{ }^{\prime}+\tau_{34}{ }^{\prime}
\end{array}
$$

where $\sigma_{i i}{ }^{\prime}, \tau_{i j}{ }^{\prime} \geqq 0$ for all $i, j$. Hence

$$
f(x)=\left(6 \sigma_{13}{ }^{\prime}+4 \sigma_{i 4}{ }^{\prime}\right) \sum_{i=1}^{4} X_{i}^{2}+\sum_{\substack{1 \leq i<j \leq 4 \\(i, j) \neq(1,3),(1,4)}} \sigma_{i j}{ }^{\prime}\left(X_{i}-X_{j}\right)^{2}+\sum_{1 \leq i<i \leq 4} \tau_{i j}{ }^{\prime}\left(X_{i}+X_{i}\right)^{2},
$$

and so, by (4.2), we may write 


$$
f(x)=\mu \omega_{1}(x)+\sum_{1 \leqq i<j \leqq 4} \sigma_{i j}\left(X_{i}-X_{i}\right)^{2}+\sum_{1 \leqq i<j \leqq 4} \tau_{i j}\left(X_{i}+X_{j}\right)^{2}
$$

where $\mu \geqq 0 ; \sigma_{i j}, \tau_{i j} \geqq 0$ for all $i, j ; \sigma_{13}=\sigma_{14}=0$.

A change in the sign of $X_{2}$ and the transformation $U$ both transform $f$ to another form of the type (4.3) with $\sigma_{13}=\sigma_{14}=0$. Furthermore, the three edge-forms $\left(X_{1}-X_{2}\right)^{2},\left(X_{1}+X_{2}\right)^{2}$ and $\left(X_{3}+X_{4}\right)^{2}$ are equivalent under these two automorphisms. Hence, we may take $f$ in the form (4.3) with $\sigma_{13}=\sigma_{14}=0$ and

$$
\tau_{34}=\min \left\{\sigma_{12}, \tau_{12}, \tau_{34}\right\} .
$$

We now split cases:

I. Suppose $\tau_{34} \leqq \sigma_{34}$. Then $\tau_{34}=\min \left\{\sigma_{12}, \tau_{12}, \sigma_{34}, \tau_{34}\right\}$ and we may subtract the term

$$
\begin{aligned}
\tau_{34}\left\{\left(X_{1}-X_{2}\right)^{2}+\left(X_{1}+X_{2}\right)^{2}+\left(X_{3}-X_{4}\right)^{2}+\left(X_{3}+X_{4}\right)^{2}\right\} & =2 \tau_{34} \sum_{i=1}^{4} X_{i}^{2} \\
& =2 \tau_{34} \omega_{1}(x)
\end{aligned}
$$

and get $f$ in the form (4.3) with $\sigma_{13}=\sigma_{14}=\tau_{34}=0$.

II. Suppose $\sigma_{34} \leqq \tau_{34}$. Then $\sigma_{34}=\min \left\{\sigma_{12}, \tau_{12}, \sigma_{34}, \tau_{34}\right\}$ and we may subtract the term

$$
\sigma_{34}\left\{\left(X_{1}-X_{2}\right)^{2}+\left(X_{1}+X_{2}\right)^{2}+\left(X_{3}-X_{4}\right)^{2}+\left(X_{3}+X_{4}\right)^{2}\right\}=2 \sigma_{34} \omega_{1}(x)
$$

and get $f$ in the form (4.3) with $\sigma_{13}=\sigma_{14}=\sigma_{34}=0$.

The two cases correspond precisely to forms $f$ belonging to the cones $\Delta^{\prime}$ and $\Delta^{\prime \prime}$ respectively of the second reduction method of Voronoi $(1908,1909)$; the above is a simpler method of obtaining the same reduction. Before we proceed to specify fundamental subregions of $\Delta^{\prime}$ and $\Delta^{\prime \prime}$, we calculate the orders of the automorphism groups of $\Delta^{\prime}$ and $\Delta^{\prime \prime}$.

Voronoi (1907, §§34-38 and $\S 43)$ examined the facets of $R\left(\varphi_{1}\right)$, that is, the 9-dimensional faces of $R\left(\varphi_{1}\right)$ in the 10-dimensional coefficient space of quaternary quadratic forms. He proved the existence of exactly 64 facets. Of these, 48 are equivalent under automorphisms of $R\left(\varphi_{1}\right)$ to the set of forms $f$ of $R\left(\varphi_{1}\right)$ in the form (4.1) with $\sigma_{13}=\sigma_{14}=\tau_{34}=0$, and the remaining 16 equivalent to the set of forms with $\sigma_{13}=\sigma_{14}=\sigma_{34}=0$. The latter 16 facets are not equivalent to the other 48 ; for the difference between any two linear forms corresponding to zero coefficients in the expansion of $f$ for a form of the second facet is of the type

$$
\left(X_{a}-X_{b}\right)-\left(X_{a}-X_{c}\right)=-\left(X_{b}-X_{c}\right)
$$

which is a linear form corresponding to a zero coefficient, whereas for a form $f$ of the first facet 


$$
\left(X_{1}-X_{3}\right)-\left(X_{1}-X_{4}\right)=-\left(X_{3}-X_{4}\right)
$$

which is not a linear form corresponding to a zero coefficient. So the 64 facets fall into two equivalence classes, the first containing $\mathbf{4 8}$ facets and the second 16 facets.

Now, the region $\Delta^{\prime}$ is the set of forms $f$ as in (4.3) with $\sigma_{13}=\sigma_{14}=\tau_{34}=0$. So it is the convex cone spanned by the form $\omega_{1}(x)$ and a facet of $R\left(\varphi_{1}\right)$ of the first kind. Since there are 48 such facets, the group $\mathscr{A}\left(\Delta^{\prime}\right)$ of automorphisms of $\Delta^{\prime}$ (including $\pm I$ ) is a subgroup of $\mathscr{A}\left(\varphi_{1}\right)$ of index 48 and so has order $3 \cdot 2^{4} \cdot 4 ! / 48=4$ !. Similarly, the region $\Delta^{\prime \prime}$ is the cone spanned by $\omega_{1}(x)$ and a facet of the second kind. Hence the group $\mathscr{A}\left(\Delta^{\prime \prime}\right)$ of automorphisms of $\Delta^{\prime \prime}$ is a subgroup of $\mathscr{A}\left(\varphi_{1}\right)$ of index 16 and so has order $3 \cdot 2^{4} \cdot 4 ! / 16=3 \cdot 4 !$.

We now consider the two cases separately.

I. Reduction of $\Delta^{\prime}$. Since the group $\mathscr{A}\left(\Delta^{\prime}\right)$ of automorphisms of $\Delta^{\prime}$ is the group of automorphisms of $R\left(\varphi_{1}\right)$ which preserve the property $\sigma_{13}=\sigma_{14}=\tau_{34}=$ 0 , clearly $\mathscr{A}\left(\Delta^{\prime}\right)$ contains

(i) the permutations of $-X_{1}, X_{3}$ and $X_{4}$

(ii) $X_{2} \mapsto-X_{2}$

and

(iii) $\left(X_{1}, X_{3}, X_{4}\right) \mapsto\left(-X_{1},-X_{3},-X_{4}\right)$.

The group generated by these automorphisms has order $3 ! \cdot 2 \cdot 2=4$ !, which is precisely the order of $\mathscr{A}\left(\Delta^{\prime}\right)$; hence the group generated is $\mathscr{A}\left(\Delta^{\prime}\right)$.

Let $f$ be a form in $\Delta^{\prime}$. By permuting $-X_{1}, X_{3}$ and $X_{4}$, we may arrange that

$$
\sigma_{34}=\min \left\{\tau_{13}, \tau_{14}, \sigma_{34}\right\} .
$$

We may still apply a permutation of $X_{3}$ and $X_{4}$, and the sign changes in (iii); so we insist further that

and

$$
\tau_{12}=\min \left\{\sigma_{12}, \tau_{12}\right\}
$$

$$
\tau_{23}=\min \left\{\tau_{23}, \tau_{24}\right\}
$$

Thus each form in $\Delta^{\prime}$ is equivalent to a form $f$ as in (4.3) with

$$
\begin{aligned}
& \mu \geqq 0 ; \sigma_{i j}, \tau_{i j} \geqq 0 \text { for all } i, j \\
& \sigma_{13}=\sigma_{14}=\tau_{34}=0 \\
& \sigma_{34}=\min \left\{\tau_{13}, \tau_{14}, \sigma_{34}\right\} \\
& \tau_{12}=\min \left\{\sigma_{12}, \tau_{12}\right\} \\
& \tau_{23}=\min \left\{\tau_{23}, \tau_{24}\right\} .
\end{aligned}
$$


By its method of specification, it is evident that the set of such forms is a fundamental region for forms of $\Delta^{\prime}$; we denote it by $F^{\prime}$. It is convex polyhedral cone with edge-forms

$$
\begin{array}{ll}
\quad \omega_{1}(x) & =\omega_{1}(x) \\
\left(X_{1}-X_{2}\right)^{2}+\left(X_{1}+X_{2}\right)^{2} & =x_{1}^{2}+x_{2}^{2} \\
\left(X_{1}-X_{2}\right)^{2} & =x_{2}^{2} \\
\left(X_{1}+X_{3}\right)^{2} & =x_{3}^{2} \\
\left(X_{1}+X_{4}\right)^{2} & =x_{4}^{2} \\
\left(X_{2}-X_{3}\right)^{2} & =\left(x_{1}-x_{3}\right)^{2} \\
\left(X_{2}-X_{4}\right)^{2} & =\left(x_{1}-x_{4}\right)^{2} \\
\left(X_{2}+X_{3}\right)^{2}+\left(X_{2}+X_{4}\right)^{2} & =\left(x_{2}-x_{3}\right)^{2}+\left(x_{2}-x_{4}\right)^{2} \\
\left(X_{2}+X_{4}\right)^{2} & =\left(x_{2}-x_{4}\right)^{2} \\
\left(X_{1}+X_{3}\right)^{2}+\left(X_{1}+X_{4}\right)^{2}+\left(X_{3}-X_{4}\right)^{2} & =x_{3}^{2}+x_{4}^{2}+\left(x_{3}-x_{4}\right)^{2} .
\end{array}
$$

Clearly, $F$ and $F^{\prime}$ have a common facet.

II. Reduction of $\Delta^{\prime \prime}$. We recall that $\Delta^{\prime \prime}$ is the set of forms $f$ as in (4.3) with $\sigma_{13}=\sigma_{14}=\sigma_{34}=0$. The group $\mathscr{A}\left(\Delta^{\prime \prime}\right)$ of automorphisms of $\Delta^{\prime \prime}$ contains

(i) the permutations of $X_{1}, X_{3}$ and $X_{4}$

(ii) $X_{2} \mapsto-X_{2}$

(iii) $\left(X_{1}, X_{3}, X_{4}\right) \mapsto\left(-X_{1},-X_{3},-X_{4}\right)$

and

(iv) the automorphism $T_{0}$ which is obtained by applying firstly the automorphism

$$
U: 2 X_{i} \mapsto 2 X_{i}-\sum_{j=1}^{4} X_{i} \quad \text { for } i=1,2,3,4,
$$

and then the automorphism

$$
\left(X_{1}, X_{2}, X_{3}, X_{4}\right) \mapsto\left(X_{4},-X_{2}, X_{1}, X_{3}\right) .
$$

Since $T_{0}$ may be shown to have order three, it is easily verified that the group generated by these automorphisms has order $3 ! \cdot 2 \cdot 2 \cdot 3=3 \cdot 4$ ! which is the order of $\mathscr{A}\left(\Delta^{\prime \prime}\right)$ (including $\pm I$ ). So the group generated is $\mathscr{A}\left(\Delta^{\prime \prime}\right)$.

Under $T_{0}$, the forms $\left(X_{i} \pm X_{j}\right)^{2}$ fall into four equivalence classes of three; the corresponding coefficients are

$$
\left\{\sigma_{13}, \sigma_{14}, \sigma_{34}\right\},\left\{\sigma_{12}, \tau_{13}, \tau_{23}\right\},\left\{\tau_{12}, \tau_{14}, \sigma_{24}\right\} \text { and }\left\{\sigma_{23}, \tau_{24}, \tau_{34}\right\}
$$


So we may arrange that the least coefficient of those in the last three classes is one of $\tau_{13}, \tau_{14}$ and $\tau_{34}$. Then, by permuting $X_{1}, X_{3}$ and $X_{4}$, we may insist that $\tau_{34}$ is the least such coefficient, that is,

$$
\tau_{34}=\min \left\{\sigma_{12}, \tau_{12}, \tau_{13}, \tau_{14}, \sigma_{23}, \tau_{23}, \sigma_{24}, \tau_{24}, \tau_{34}\right\} .
$$

Since $\mathscr{A}\left(\Delta^{\prime \prime}\right)$ includes both $\pm I$, we may arbitrarily fix the sign of $X_{1}$, and so by (iii) of $X_{3}$ and $X_{4}$. Using (ii), we may now specify that

$$
\tau_{12}=\min \left\{\sigma_{12}, \tau_{12}\right\} .
$$

The only automorphism not yet used is the interchange of $X_{3}$ and $X_{4}$; so we may now insist that

$$
\tau_{23}=\min \left\{\tau_{23}, \tau_{24}\right\}
$$

It is evident that the set of forms $f$ as in (4.3) with $\sigma_{13}=\sigma_{14}=\sigma_{34}=0$ satisfying (4.4), (4.5) and (4.6) is a fundamental region for those forms in $\Delta^{\prime \prime}$; let us denote it by $F^{\prime \prime}$. It is a convex polyhedral cone with edge-forms

$$
\begin{array}{ll}
\quad \omega_{1}(x) & =\omega_{1}(x) \\
\left(X_{1}-X_{2}\right)^{2}+\left(X_{1}+X_{2}\right)^{2} & =x_{1}^{2}+x_{2}^{2} \\
\left(X_{1}-X_{2}\right)^{2} & =x_{2}^{2} \\
\left(X_{1}+X_{3}\right)^{2} & =x_{3}^{2} \\
\left(X_{1}+X_{4}\right)^{2} & =x_{4}^{2} \\
\left(X_{2}-X_{3}\right)^{2} & =\left(x_{1}-x_{3}\right)^{2} \\
\left(X_{2}-X_{4}\right)^{2} & =\left(x_{1}-x_{4}\right)^{2} \\
\left(X_{2}+X_{3}\right)^{2}+\left(X_{2}+X_{4}\right)^{2} & =\left(x_{2}-x_{3}\right)^{2}+\left(x_{2}-x_{4}\right)^{2} \\
\left(X_{2}+X_{4}\right)^{2} & =\left(x_{2}-x_{4}\right)^{2} \\
\left(X_{1}-X_{2}\right)^{2}+\left(X_{1}+X_{2}\right)^{2}+\left(X_{1}+X_{3}\right)^{2}= & x_{1}^{2}+x_{2}^{2}+x_{3}^{2}+x_{4}^{2} \\
+\left(X_{1}+X_{4}\right)^{2}+\left(X_{2}-X_{3}\right)^{2} & \quad+\left(x_{1}-x_{3}\right)^{2}+\left(x_{1}-x_{4}\right)^{2} \\
+\left(X_{2}+X_{3}\right)^{2}+\left(X_{2}-X_{4}\right)^{2} & \quad+\left(x_{2}-x_{3}\right)^{2}+\left(x_{2}-x_{4}\right)^{2} \\
+\left(X_{2}+X_{4}\right)^{2}+\left(X_{3}+X_{4}\right)^{2} & \quad+\left(x_{1}+x_{2}-x_{3}-x_{4}\right)^{2} .
\end{array}
$$

By comparison, we see that $F^{\prime}$ and $F^{\prime \prime}$ have a facet in common, while $F$ and $F^{\prime \prime}$ have a common 8-dimensional face. 


\section{Union of the reduced regions}

The region of forms given in $\$ 1$ to be proved fundamental is the convex hull $H$ of $F \cup F^{\prime} \cup F^{\prime \prime}$. Since every positive definite quaternary quadratic form is equivalent to a form of $R\left(\varphi_{0}\right)$ or $R\left(\varphi_{1}\right)$, every such form is equivalent to one of $F \cup F^{\prime} \cup F^{\prime \prime}$ and hence of $H$. To show that $H$ is fundamental, we show that $F \cup F^{\prime} \cup F^{\prime \prime}$ is precisely $H$ and that the region $H$ has no two equivalent forms in its interior.

Firstly, we show that $F \cup F^{\prime} \cup F^{\prime \prime}$ is $H$. Since $F \cup F^{\prime} \cup F^{\prime \prime}$ is a subset of $H$, it is sufficient to show that $H$ is a subset of $F \cup F^{\prime} \cup F^{\prime \prime}$. Now $H$ is a convex cone with at most twelve edges. Eight edges are common to $F, F^{\prime}$ and $F^{\prime \prime}$; the other four are generated by the forms

$$
x_{3}^{2}+x_{4}^{2}+\left(x_{3}-x_{4}\right)^{2}, \quad \omega_{0}(x), \omega_{1}(x)
$$

and

$$
\begin{aligned}
\alpha(x)=x_{1}^{2} & +x_{2}^{2}+x_{3}^{2}+x_{4}^{2}+\left(x_{1}-x_{3}\right)^{2}+\left(x_{1}-x_{4}\right)^{2} \\
& +\left(x_{2}-x_{3}\right)^{2}+\left(x_{2}-x_{4}\right)^{2}+\left(x_{1}+x_{2}-x_{3}-x_{4}\right)^{2} .
\end{aligned}
$$

It is easily established that

$$
\begin{aligned}
& 2 \omega_{0}(x)+\omega_{1}(x) \in F \cap F^{\prime}, \\
& \left\{x_{3}^{2}+x_{4}^{2}+\left(x_{3}-x_{4}\right)^{2}\right\}+\alpha(x) \in F^{\prime} \cap F^{\prime \prime} \\
& \omega_{0}(x)+\alpha(x) \in F \cap F^{\prime} \cap F^{\prime \prime} .
\end{aligned}
$$

and

(The argument also shows that no edge-form of $H$ is redundant, and so $H$ has precisely twelve edges.) Hence, since any form of $H$ may be expressed as a nonnegative linear combination of the twelve edge-forms of $H$, by considering the relative magnitudes of the coefficients corresponding to the above four edge-forms, we may deduce that a form of $H$ belongs to one of $F, F^{\prime}$ and $F^{\prime \prime}$, and so to $F \cup F^{\prime} \cup F^{\prime \prime}$.

Finally, we prove that no two distinct forms in the interior of $H$ are equivalent. For suppose $f, g$ are distinct interior forms of $H$ with $f \sim g$. Then $f, g$ are equivalent by a continuous transformation of the space of forms. So there exist equivalent forms $f_{0}, g_{0}$ arbitrarily close to $f, g$ respectively for which $f_{0} \neq g_{0}$ and either

(i) both $f_{0}$ and $g_{0}$, belong to the interior of the same one of $F, F^{\prime}$ and $F^{\prime \prime}$ or

(ii) $f_{0}$ and $g_{0}$ belong to the interiors of different sets of $F, F^{\prime}$ and $F^{\prime \prime}$. Both situations are impossible, since $F, F^{\prime}$ and $F^{\prime \prime}$ are fundamental regions for forms of $\Delta\left(=R\left(\varphi_{0}\right)\right), \Delta^{\prime}$ and $\Delta^{\prime \prime}$ respectively. 


\section{References}

H. S. M. Coxeter (1951), 'Extreme forms', Canad. J. Math. 3, 391-441.

H. Minkowski (1905), 'Diskontinuitätsbereich für arithmetische Äquivalenz', J. reine angew. Math. 129, 220-274.

M. I. Štogrin (1974), 'On the classification of 4-dimensional lattices according to Bravais, Voronoi and Delone' (Russian), Dokl. Akad. Nauk SSSR 218, 528-531.

B. A. Venkov (1940), 'On the reduction of positive quadratic forms' (Russian), Izv. Akad. Nauk SSSR Ser. Mat. 4, 37-52.

G. Voronoi (1907), 'Sur quelques propriétés des formes quadratiques positives parfaites', J. reine angew. Math. 133, 97-178.

G. Voronoi (1908), 'Recherches sur les paralléloèdres primitifs' (Part 1), J. reine angew. Math. 134, 198-287.

G. Voronoi (1909), 'Recherches sur les paralléloèdres primitifs' (Part 2), J. reine angew. Math. 136, 67-181.

University of Adelaide

Adelaide

South Australia 5001. 